\title{
Avionics System Architecture for NASA Orion Vehicle
}

\author{
Clint Baggerman \\ NASA- Johnson Space Center
}

TTA Group Open Forum

November $4^{\text {th }}, 2010$ 


\section{What is Orion?}

- The Orion Crew Exploration Vehicle will provide a capability to deliver humans to space reliably and return them safely

- Orion is currently under development by NASA, Lockheed Martin, and other industry partner

- The first Orion spaceflight will be an uncrewed flight test

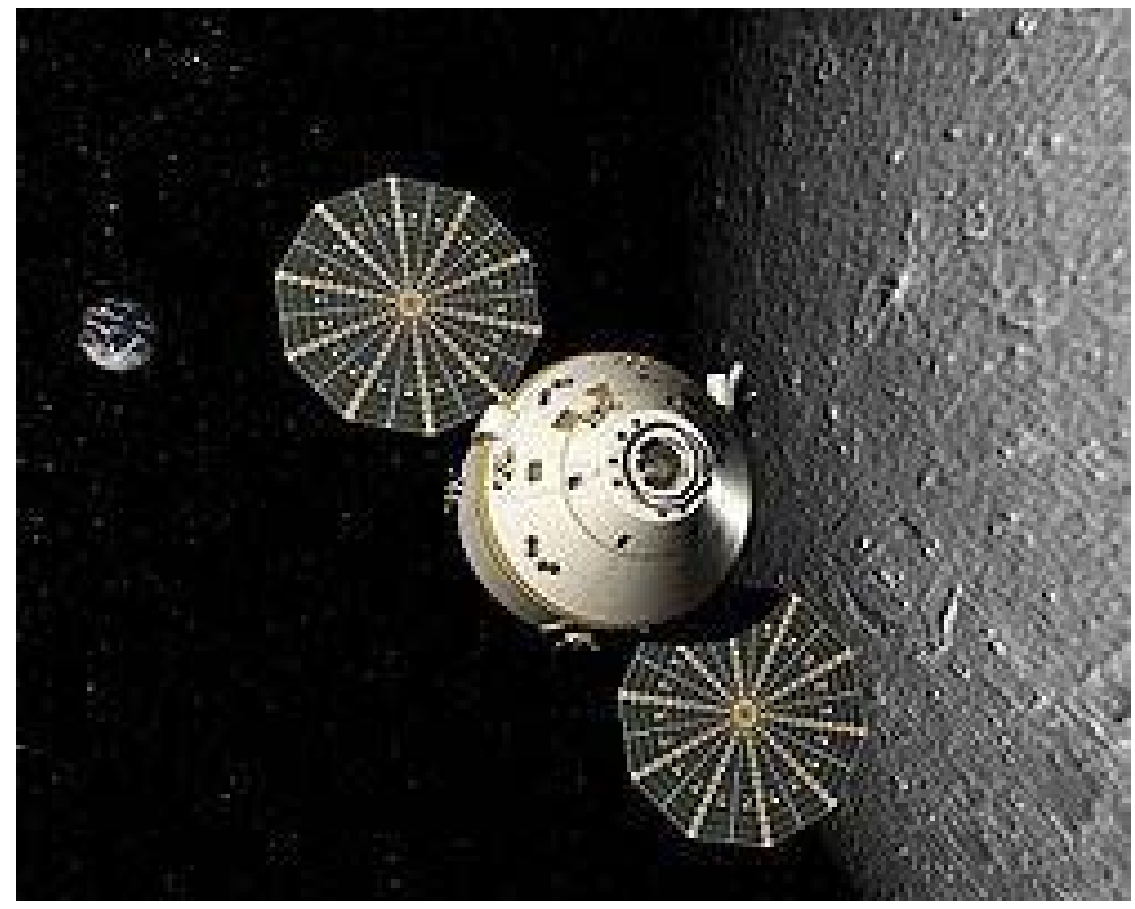


Nasa Orion Concept of Operations

- Potential Orion mission objectives include delivering a crew to the International Space Station, transporting a crew to a near-Earth objects, and providing emergency return capability from the International Space Station

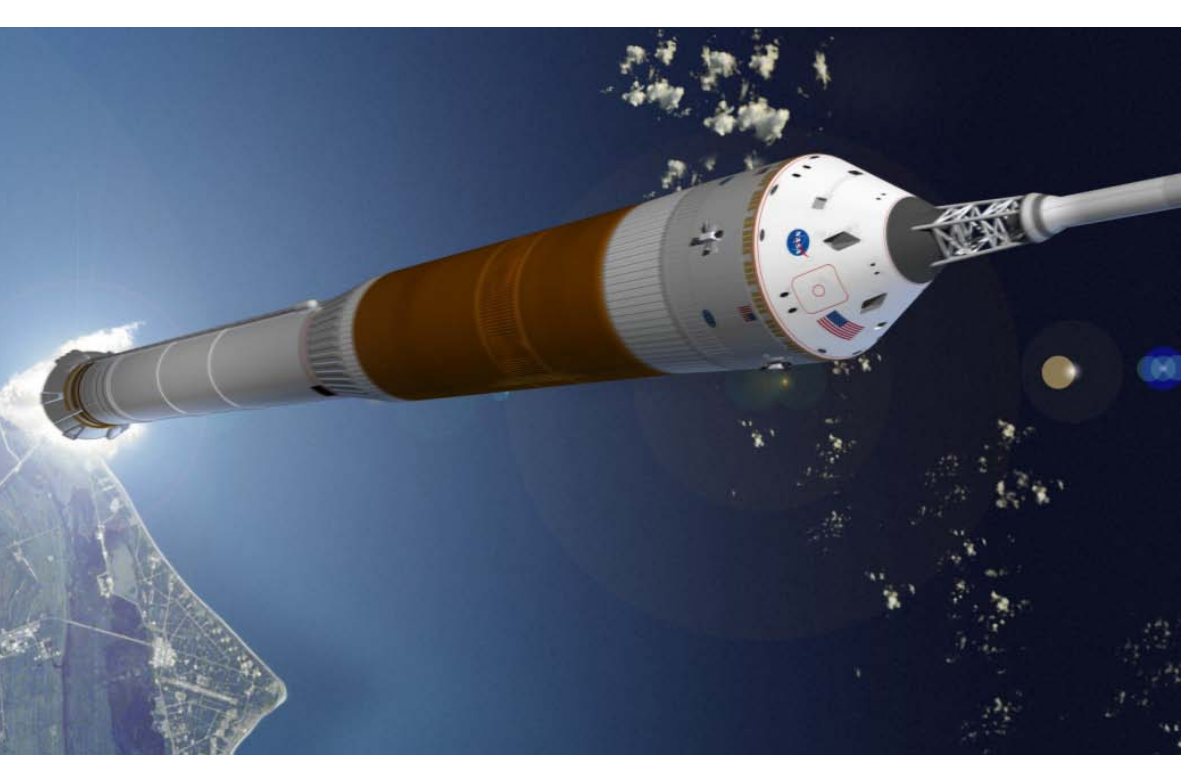

- Crew of 4

- Crew launch from Kennedy Space Center

- Ocean landing off California coast

- Ability to abort during launch 


\section{Orion Subsystems}

- Orion contains the following vehicle subsystems:

- Propulsion

- Vehicle power

- Life support

- Communications

- Docking adapter

- Structures

- Pyrotechnics
- Displays and Controls

- Parachutes

- Guidance \& Navigation

- Mechanisms

- Crew Systems

- Thermal Control

- Thermal Protection

- The Orion Avionics subsystem must provide an infrastructure to command, control, and monitor all of these subsystems 


\section{Orion Avionics Architecture}

- Orion uses an IMA-based high integrity architecture with the following elements:

- Vehicle Management Computers (VMCs)

- Provides a central computing platform to host software applications for a variety of vehicle subsystems

- Time-Triggered Ethernet (TTEthernet) Onboard Data Network

- Provides priority-based network communications via time triggered, rate constrained, and best effort traffic classes

- Power and Data Units (PDUs)

- Provides sensor data gathering, actuator control, and power distribution for critical vehicle subsystems 


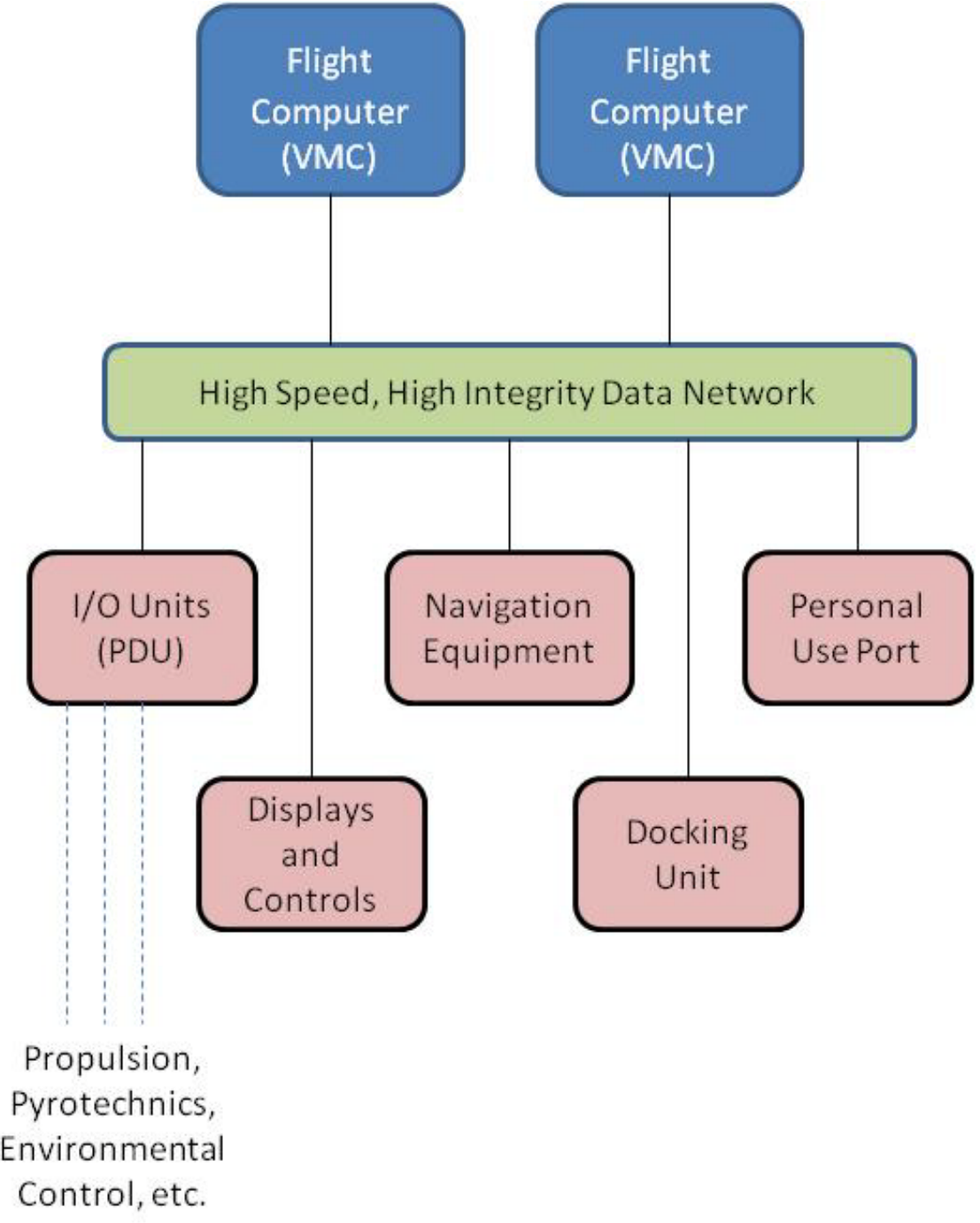




\section{Orion Avionics- Network}

- Orion uses Time Triggered Ethernet (TTEthernet) to provide high-integrity, deterministic data network communications across the vehicle

- The data network is deterministic to guarantee latency and response time for critical sensors and effectors

- Traffic classes (time triggered, rate constrained, and best effort) allow prioritization of network data

- Cross-comparison of data provides fault containment at the network switches and safety critical interfaces 


\section{Orion Network Unification}

- The original Orion architecture contained two Ethernet-based data networks:

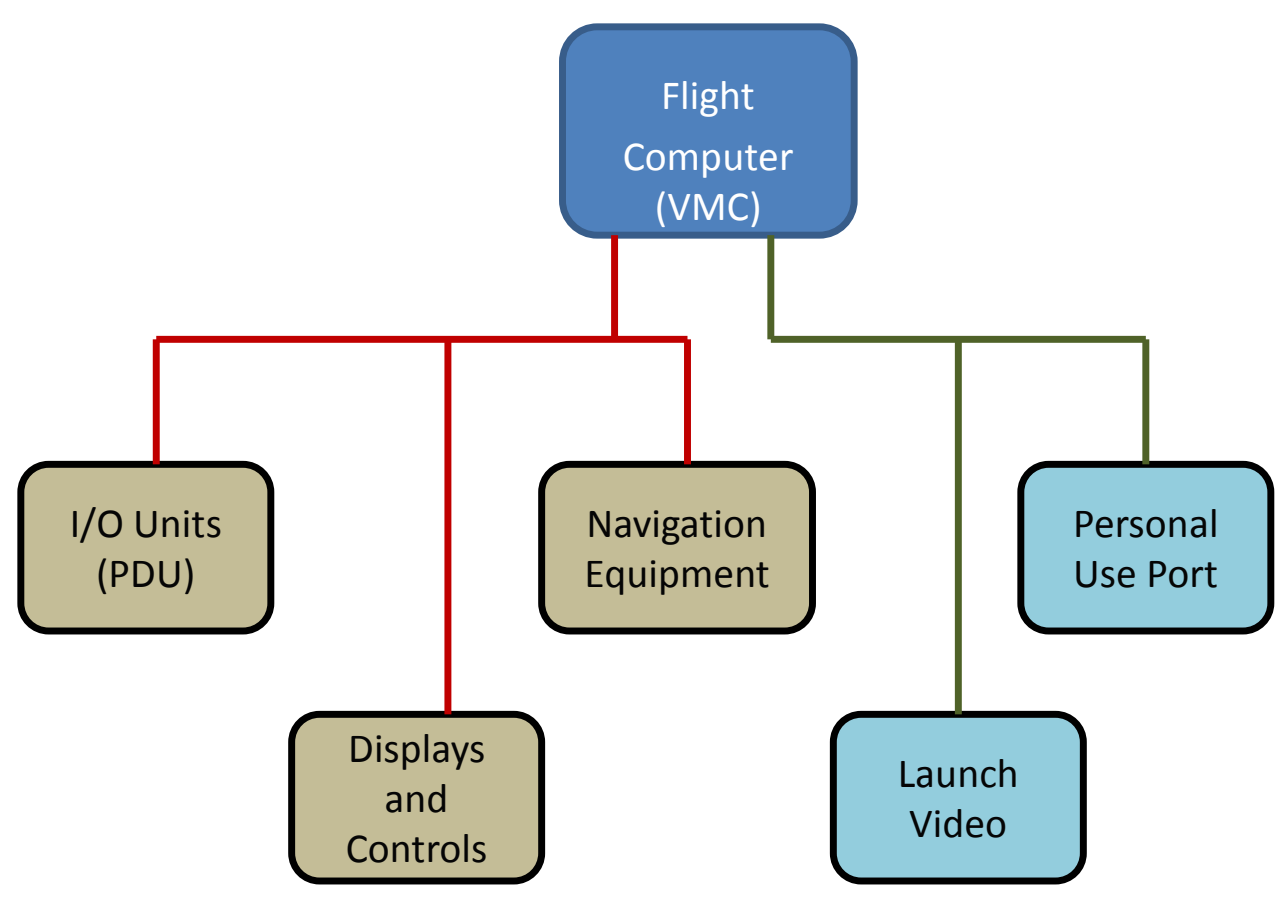

- A flight-critical control bus that handled time-sensitive and/or safety critical commands

- A general-purpose data bus that handled non-critical traffic such as video and personal crew equipment 


\section{Orion Network Unification}

- However, to reduce vehicle size, weight, and power while maintaining acceptable reliability, Orion collapsed both networks into one, TTEthernet-based infrastructure

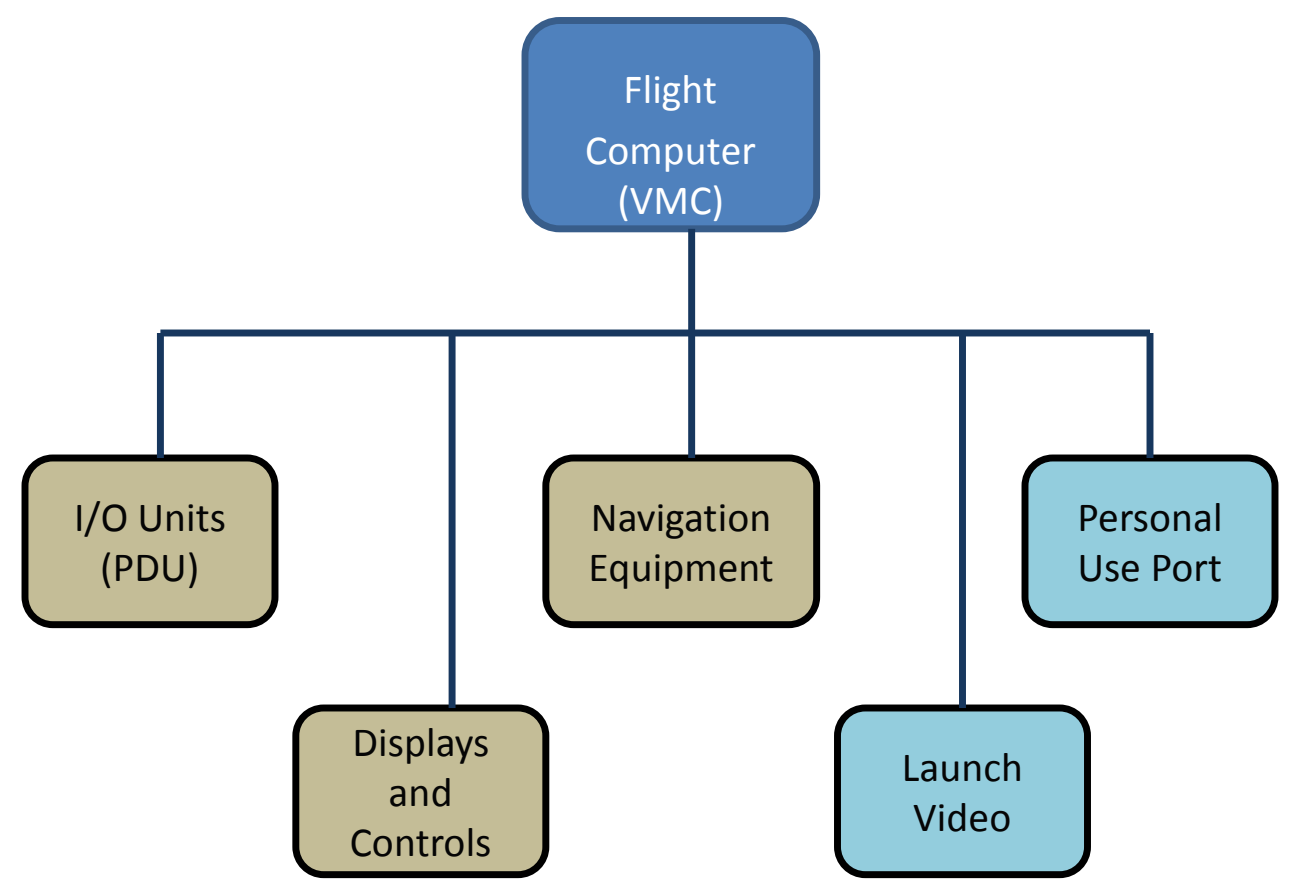

- Critical or time-sensitive data utilized time-triggered or rate-constrained TTEthernet traffic classes

- Video and personal crew data utilized the best effort TTEthernet traffic class 
- Orion VMCs utilize a self-checking pair of processors to ensure the integrity of commands issued to vehicle subsystems

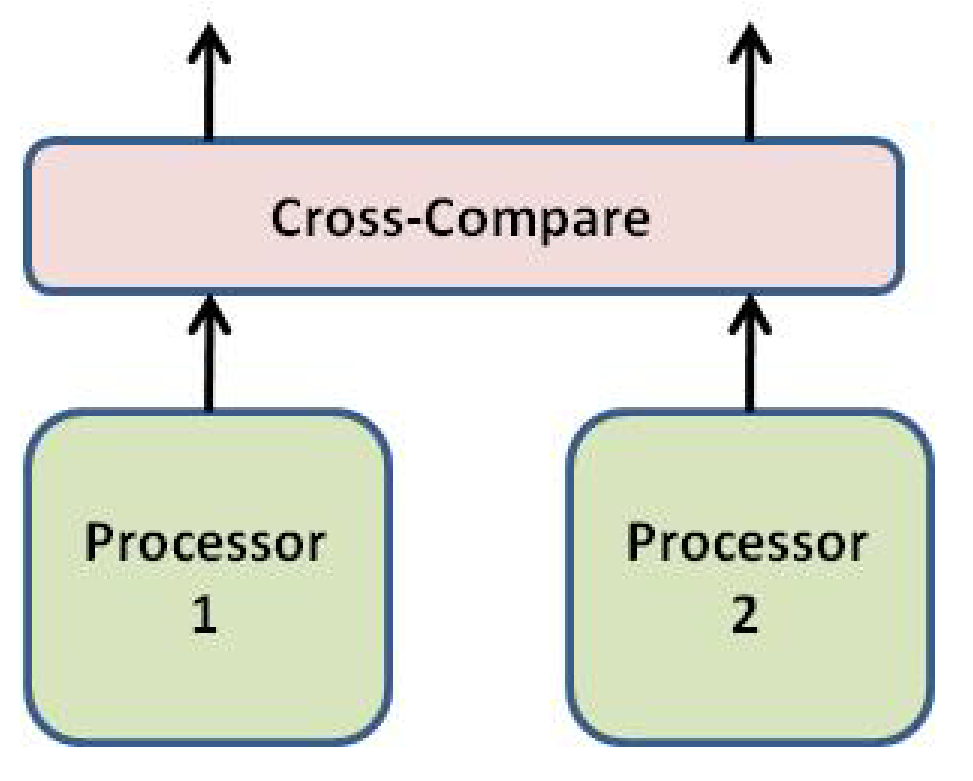

- Each VMC contains two processors - Each processor independently runs the same applications

- The processor outputs are bit-by-bit compared to one another

- If any miscompares are detected between the outputs, that command is truncated 
- Orion VMCs utilize time and space partitioning of software and memory to ensure faults do not propagate between systems

- VMCs execute code for a variety of software applications supporting various vehicle systems

- Code for each software application is located in a unique, specific memory space

- Each software application runs during a specific, periodic slice of time

- As a result, faults from one system will not threaten the execution of other

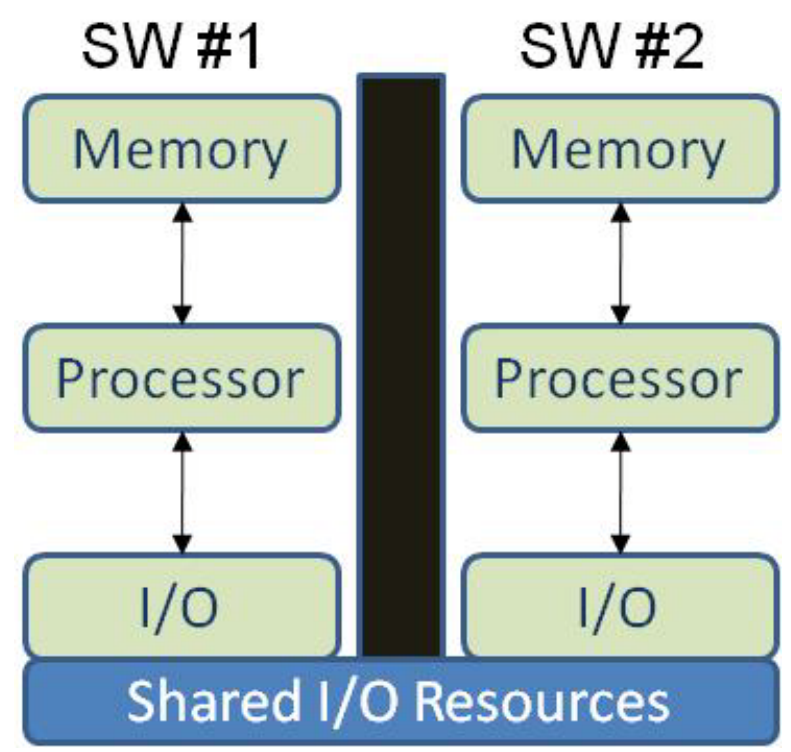
systems' code 
- Orion avionics uses simple redundancy (minimum one fault tolerance) to ensure that commands are successfully received

- A command follows the following sequence:

- A command is generated on each VMC

- It is then sent simultaneously over two plane of the Data Network

- The valid command is received from both VMCs at each of two PDUs

- The PDUs send control signals initiated by the commands to redundant effectors 